\title{
DETERMINAÇÃO SIMULTÂNEA DE ÁCIDO ASCÓRBICO E ÁCIDO ACETILSALICÍLICO USANDO ANÁLISE POR INJEÇÃO EM FLUXO COM DETECÇÃO AMPEROMÉTRICA PULSADA
}

\author{
Joyce A. T. de Miranda, Rafael R. Cunha, Denise T. Gimenes, Rodrigo A. A. Munoz e Eduardo M. Richter* \\ Instituto de Química, Universidade Federal de Uberlândia, Av. João Naves de Ávila, 2121, 38400-902 Uberlândia - MG, Brasil
}

Recebido em 22/11/11; aceito em 28/1/12; publicado na web em 30/4/12

\begin{abstract}
SIMULTANEOUS DETERMINATION OF ASCORBIC ACID AND ACETYLSALICYLIC ACID USING FLOW INJECTION ANALYSIS WITH MULTIPLE PULSE AMPEROMETRIC DETECTION. A simple flow system with multiple pulse amperometric detection using a single working electrode is proposed for simultaneous determination of ascorbic (AA) and acetylsalicylic (AAS) acids in pharmaceutical formulations. The procedure is based on application of two potential pulses: $0.90 \mathrm{~V} / 50 \mathrm{~ms}$ : oxidation and determination of AA without the interference of AAS; 1.35 V/50 ms: oxidation of both compounds and quantification of AAS by current subtraction using a correction factor. Sampling rate was estimated as 125 injections per hour and the limits of detection were 0.17 and $0.16 \mu \mathrm{mol} \mathrm{L}^{-1}$ for AA and AAS, respectively. Results for commercial samples agreed with those obtained using HPLC.
\end{abstract}

Keywords: simultaneous determination; FIA; multiple pulse amperometry.

\section{INTRODUÇÃO}

A aspirina ou ácido acetilsalicílico (AAS) é um dos anti-inflamatórios não esteroidais (AINE) mais usados no mundo. É indicado como analgésico, antipirético e anti-inflamatório no tratamento de febres reumáticas, crises de gota e artrites reumáticas. No entanto, o uso constante do AAS também pode gerar problemas de saúde, principalmente na indução de lesões gástricas. ${ }^{1}$ Em algumas formulações farmacêuticas disponíveis no mercado, o AAS é encontrado combinado com ácido ascórbico (AA) e, neste caso, é indicado principalmente no alívio de sintomas de gripes e resfriados. Segundo estudos realizados, quando o AAS está associado com AA, são necessárias doses menores de AAS para se atingir o mesmo benefício, com consequente diminuição na indução de efeitos colaterais provocados pelo AAS. ${ }^{2}$ Além disto, as características antioxidantes do AA também estão presentes. ${ }^{3}$

A presença dos dois princípios ativos (AA + AAS) na mesma formulação é vantajosa. Por outro lado, o controle de qualidade desta formulação se torna mais complicado, pois um número considerável de métodos analíticos usados na quantificação de AAS somente é eficiente na ausência de AA, que é considerado um interferente na determinação de AAS em grande parte dos métodos disponibilizados na literatura. ${ }^{4}$ Considerando sua ampla utilização e os possíveis efeitos colaterais, é de grande relevância o desenvolvimento de métodos analíticos que permitam a determinação simultânea de AAS e AA. Entre os métodos propostos que permitem essa determinação simultânea destacam-se os cromatográficos ${ }^{5}$ e os espectrofotométricos. ${ }^{6}$ Segundo nosso conhecimento, não existe na literatura um trabalho com a descrição de um método que possibilite a análise simultânea de AAS e AA empregando detecção eletroquímica (voltametria ou amperometria).

Diamante dopado com boro (BDD - boron-doped diamond) é um material eletródico relativamente novo ${ }^{7}$ e que tem se destacado em diversas aplicações, devido a suas propriedades eletroquímicas, como corrente de fundo baixa e estável, estabilidade à corrosão em meios quimicamente agressivos, fraca adsorção de moléculas polares, o que melhora a resistência do eletrodo à desativação ou envenenamento, estabilidade de resposta em longo prazo, larga janela de potencial

*e-mail: emrichter@iqufu.ufu.br elétrico e alta sensibilidade. Informações mais detalhadas sobre este material podem ser localizadas em artigos de revisão sobre o tema. ${ }^{8}$ Provavelmente, as características singulares do BDD foram responsáveis pela preferência por este material no desenvolvimento de métodos de controle de qualidade de diversos princípios ativos presentes em formulações farmacêuticas. ${ }^{9}$

A análise por injeção em fluxo com detecção amperométrica de múltiplos pulsos (FIA-MPA, flow injection analysis and multiple pulse amperometry ${ }^{10}$ é um procedimento de análise que vem sendo explorado na determinação simultânea de compostos empregando um único eletrodo de trabalho, tanto por FIA, ${ }^{11}$ como por análise por injeção em batelada (BIA, batch injection analysis). ${ }^{12}$ Além de permitir determinações simultâneas, este procedimento também propicia a obtenção de melhor seletividade ${ }^{13}$ e precisão, mediante a possibilidade da inclusão de um pulso de potencial que promove limpeza eletroquímica constante ${ }^{14}$ ou de empregar o método do padrão interno. ${ }^{15}$

Neste trabalho apresenta-se um procedimento simples usando FIA com detecção amperométrica de múltiplos pulsos (MPA) para a determinação simultânea de ácido ascórbico (AA) e ácido acetilsalicílico (AAS) em formulações farmacêuticas.

\section{PARTE EXPERIMENTAL}

\section{Reagentes, soluções e amostras}

Todas as soluções foram preparadas com água deionizada (Gehaka-Master-System, modelo OS 20LX) com resistividade igual ou superior a $18 \mathrm{M} \Omega-\mathrm{cm}$. Ácido acético $(65 \% \mathrm{~m} / \mathrm{v})$ e hidróxido de sódio sem purificação prévia foram usados na preparação da solução tampão de ácido acético/acetato $0,1 \mathrm{~mol} \mathrm{~L}^{-1}(\mathrm{pH}=4,7)$. Soluções padrão de AA e AAS (Synth, Diadema, Brasil) foram preparadas imediatamente antes do uso, através de diluições apropriadas das soluções estoque. As amostras e soluções estoque contendo AAS foram preparadas em meio de $\mathrm{NaOH} 0,2 \mathrm{~mol} \mathrm{~L}^{-1}$ para conversão por hidrólise do acetilsalicilato em salicilato $(15 \mathrm{~min}) .{ }^{16}$ As amostras foram preparadas seguindo o seguinte procedimento: 12 comprimidos de um determinado lote foram macerados e em torno de $0,144 \mathrm{~g}$ foram transferidos para um balão volumétrico de $10 \mathrm{~mL}$ e o volume foi completado com uma solução de hidróxido de sódio 0,2 mol L-1. 
Alíquotas desta solução foram posteriormente diluídas na solução usada como tampão e eletrólito.

A fase móvel usada na determinação de AAS e AA por HPLC era composta de uma mistura de uma solução aquosa de ácido fosfórico ( $\mathrm{pH}$ ajustado em 2,1 com $\mathrm{NaOH}$ ) e acetonitrila $(60 / 40 \% \mathrm{v} / \mathrm{v}$, respectivamente). As condições cromatográficas foram adaptadas a partir de um artigo em que os fármacos AAS, paracetamol, cafeína e fenobarbital foram quantificados por HPLC. ${ }^{17}$

\section{Instrumentação}

Todos os experimentos eletroquímicos empregando voltametria cíclica ou amperometria de múltiplos pulsos foram realizados usando um potenciostato $\mu$ Autolab Type III interfaceado a um computador através do software GPES (versão 4.9.007). O sistema FIA usado é de linha única empregando um injetor comutador manual (CENAUSP $)^{18}$ e uma célula eletroquímica do tipo wall-jet. Detalhes sobre a construção da célula eletroquímica podem ser localizados em trabalho publicado anteriormente. ${ }^{19}$ É importante salientar que a célula eletroquímica empregada permite que o procedimento de limpeza eletroquímica seja realizado com o eletrodo de trabalho (BDD) posicionado na célula em fluxo (sem necessidade de desmontagem), o que pode ser considerado uma vantagem em relação às células de camada delgada. ${ }^{20}$

Os eletrodos de referência e auxiliar foram de $\mathrm{Ag} / \mathrm{AgCl}$ (saturado em $\mathrm{KCl})^{21}$ e um fio de platina, respectivamente. Uma película fina $(\sim 1.2 \mu \mathrm{m})$ de diamante dopado com boro (BDD) depositado sobre uma placa $(7 \times 7 \mathrm{~mm})$ policristalina de silício de $1,2 \mathrm{~mm}$ de espessura foi usada como eletrodo de trabalho. $\mathrm{O}$ material foi adquirido de Adamant Technologies SA, La Chaux-de-Fonds, Suíça. Neste trabalho, dois tipos de limpezas eletroquímicas (ativações) foram realizados com o eletrodo de BDD, a anódica $(+2,6 \mathrm{~V}$, durante $900 \mathrm{~s}$ em meio de tampão Britton-Robinson $)^{22}$ e a catódica (-3,0 V durante $900 \mathrm{~s}$ em meio de $\left.\mathrm{H}_{2} \mathrm{SO}_{4} 0,2 \mathrm{~mol} \mathrm{~L}^{-1}\right)$. ${ }^{23}$ Diariamente, antes do início das análises, apenas a limpeza catódica era efetivamente realizada. A limpeza anódica somente era realizada quando a limpeza catódica não gerava resultados considerados satisfatórios (corrente de fundo < $1 \mu \mathrm{A}$ empregando-se voltametria cíclica em meio de $\mathrm{H}_{2} \mathrm{SO}_{4}$ ). Após a limpeza anódica, a limpeza catódica necessita ser repetida. A vazão do sistema FIA foi controlada por pressão gerada por uma coluna d'água e uma bomba de aquário ${ }^{24}$ ou através de uma bomba peristáltica (Minipuls 3 - Gilson) mediante o uso de um amortecedor de pulsos. Todos os estudos foram realizados em temperatura ambiente e na presença de oxigênio dissolvido.

$\mathrm{O}$ equipamento de cromatografia de líquida de alta eficiência utilizado na validação do método proposto consistiu em bomba L-2130, marca Hitachi VWR; fase estacionária coluna C18, marca Shim-PAC CLC-ODS; detector UV-VIS, marca Merck-Hitachi, com o comprimento de onda $(\lambda)$ ajustado em $210 \mathrm{~nm} .{ }^{17}$

\section{RESULTADOS E DISCUSSÃO}

Inicialmente, o comportamento eletroquímico do AA e do AS (AAS hidrolisado) foi estudado usando-se voltametria cíclica (Figura 1A) e o sistema FIA-MPA (Figura 1B). Estes estudos foram realizados com vários eletrólitos suporte, empregando-se BDD como eletrodo de trabalho $\left(\mathrm{H}_{2} \mathrm{SO}_{4}\right.$, tampão acetato - $\mathrm{pH}=4,7$ e fosfato - $\left.\mathrm{pH}=7,2\right)$. A solução tampão de ácido acético/acetato $0,1 \mathrm{~mol} \mathrm{~L}^{-1}(\mathrm{pH}=4,7)$ apresentou os melhores resultados, considerando-se características como sensibilidade, estabilidade e resolução entre os picos de oxidação do AA e AS. Estudos realizados com outros eletrodos de trabalho (ouro e carbono vítreo) não levaram a resultados satisfatórios, principalmente em relação à estabilidade de sinal em função do tempo e experimentos sucessivos (contaminação gradativa e eletroquimicamente irreversível da superfície do eletrodo).
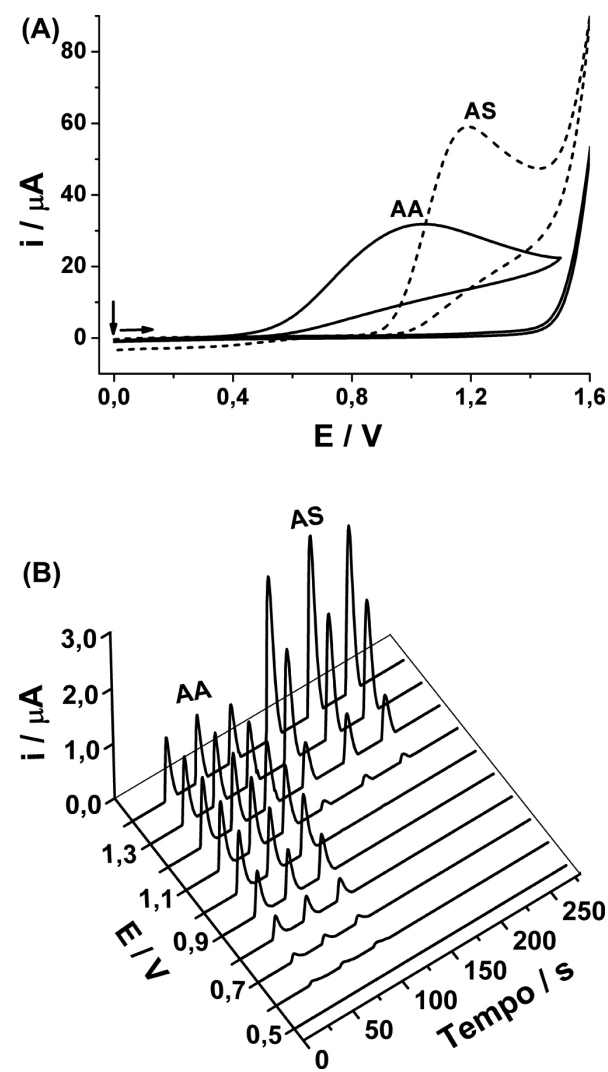

Figura 1. (A) Voltamogramas cíclicos obtidos em meio de tampão HAc/NaAc $0,1 \mathrm{~mol} \mathrm{~L}^{-1}(p H=4,7)$ sem e com a adição de 2,0 mmol $L^{-1}$ de AA ou 2,0 mmol $L^{-1}$ de AS. Velocidade de varredura de potencial: $50 \mathrm{mV} \mathrm{s}^{-1}$; incremento de potencial: $5 \mathrm{mV}$. (B) Amperogramas obtidos usando o sistema FIA-MPA na injeção de $60 \mu \mathrm{mol} \mathrm{L} L^{-1}$ de AA e $100 \mu \mathrm{mol} \mathrm{L} L^{-1}$ de AS. Vazão: 3,0 $\mathrm{mL} \mathrm{min}{ }^{-1}$; volume injetado: $100 \mu \mathrm{L}$; eletrólito: $\mathrm{HAc} / \mathrm{NaAc} 0,1 \mathrm{~mol} \mathrm{~L}^{-1}$; pulsos de potenciais aplicados: 0,5; 0,6; 0,7;0,8; 0,9;1,0; 1,1;1,2;1,3 e 1,4 V(50 ms cada)

Os resultados obtidos por voltametria cíclica e FIA-MPA foram similares. O início da oxidação das espécies estudadas, em FIAMPA, ocorreu em potenciais mais positivos, o que se deve ao uso de soluções mais diluídas no experimento. Conforme pode ser observado nas Figuras 1A e 1B, a oxidação do AA a ácido de-hidroascórbico se inicia em torno de $+0,50 \mathrm{~V}$, sendo que a corrente de pico obtida foi em torno de $+1,00 \mathrm{~V}$. No sentido inverso da varredura (VC), o AA não apresenta sinal de redução, o que caracteriza um processo irreversível nas condições experimentais empregadas. ${ }^{13}$ A oxidação do ácido salicílico (AS) a ácido di-hidróxi-benzoico se inicia em aproximadamente $+0,95 \mathrm{~V}$, apresentando corrente de pico em +1,20 V. Diferentemente do AA, o AS apresenta um sinal no sentido inverso (VC) da varredura a partir de $+0,45 \mathrm{~V}$ (redução). No entanto, a corrente de redução é diversas vezes inferior à corrente de oxidação ( 20 vezes). Segundo a literatura, este sinal de menor intensidade se deve a uma reação química acoplada ao processo de oxidação do AS. Segundo Enache et al.,${ }^{16}$ durante a oxidação do AS sobre eletrodo de BDD, são gerados radicais hidroxila no eletrodo e ocorre uma hidroxilação do composto, formando o ácido di-hidróxi-benzoico (2,3 e 2,5).

A partir dos resultados apresentados na Figura 1, dois pulsos de potenciais foram selecionados e usados no sistema FIA-MPA: +0,9 V/50 ms: oxidação e quantificação de AA sem a interferência de AS e +1,35 V/50 ms: oxidação de ambos os analitos (AA+AS).

A Figura 2 apresenta os amperogramas obtidos com a injeção no 
sistema FIA-MPA (+0,90 e 1,35 V) de uma solução contendo somente AA $\left(60 \mu \mathrm{mol} \mathrm{L}^{-1}\right)$ ou somente AS $\left(100 \mu \mathrm{mol} \mathrm{L}^{-1}\right)$ ou ambas as espécies $\left(60+100 \mu \mathrm{mol} \mathrm{L}^{-1}\right.$ de AA+AS, respectivamente).

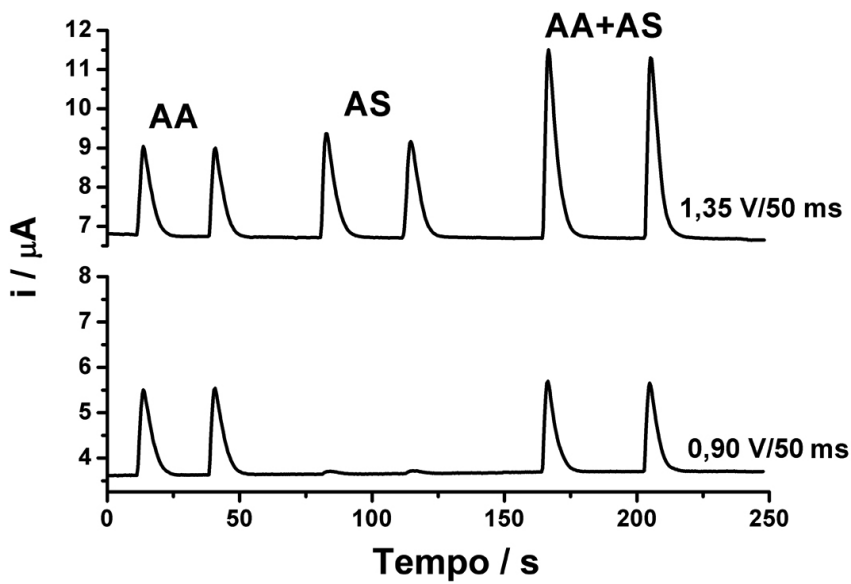

Figura 2. Amperogramas obtidos após a injeção (em duplicata) no sistema FIA-MPA de $60 \mu \mathrm{mol} \mathrm{L} L^{-1}$ de AA; $100 \mu \mathrm{mol} \mathrm{L} L^{-1}$ de AS e uma solução contendo ambas as espécies na mesma concentração anterior $(A A+A S)$. Eletrólito: HAc/NaAc 0,10 mol L $L^{-1}(p H 4,7)$; vazão: $3,5 \mathrm{~mL} \mathrm{~min}^{-1}$; volume injetado: $100 \mu \mathrm{L}$

Como era esperado e como pode ser observado na Figura 2, o AA é oxidado em ambos os pulsos de potenciais e o AS somente em $+1,35 \mathrm{~V}$. Desta forma, conclui-se que o AA pode ser detectado e quantificado no pulso de potencial de $+0,9 \mathrm{~V}$ sem a interferência do AS. O AS, por sua vez, somente é eletroativo no pulso de potencial de $1,35 \mathrm{~V}$, onde o AA também é oxidado. A estratégia proposta neste trabalho para a quantificação seletiva do AS é o uso da diferença de sinal detectada nos dois pulsos de potenciais:

$$
\mathrm{I}_{\mathrm{AS}}=\mathrm{I}_{+1,35 \mathrm{~V}}-\mathrm{I}_{+0,9 \mathrm{~V}}
$$

Porém, conforme pode ser observado na Figura 2, a corrente de oxidação do AA não apresenta a mesma magnitude em ambos os pulsos de potenciais, o que impede o uso de uma subtração simples de sinal para se ter acesso à corrente proveniente somente da oxidação do AS em 1,35 V. Uma opção adotada neste trabalho foi: soluções contendo somente AA foram injetadas para se determinar a corrente de oxidação detectada nos dois pulsos de potenciais utilizados $(+0,9$ e $+1,35 \mathrm{~V})$. A partir deste resultado experimental, um fator pode ser calculado:

$$
\text { fator }=\mathrm{I}_{+1,35 \mathrm{v}} / \mathrm{I}_{+0,9 \mathrm{~V}}
$$

Este fator foi usado para determinar a corrente proveniente da oxidação do AS no pulso de potencial de $+1,35 \mathrm{~V}$ quando uma solução contendo as duas espécies era injetada.

$$
\mathrm{I}_{\mathrm{AS}}=\mathrm{I}_{+1,35 \mathrm{~V}}-\left(\text { fator } \mathrm{x} \mathrm{I}_{+0,9 \mathrm{v}}\right)
$$

Conforme descrito na literatura, ${ }^{25}$ a oxidação do AS pode contaminar a superfície de um eletrodo de trabalho sólido usado no experimento, o que pode restringir seu uso em um método de quantificação. Em função disto, um estudo de repetibilidade foi realizado com o sistema proposto. Duas soluções contendo AA+AS $(30+50$ e $80+130 \mu \mathrm{mol} \mathrm{L}^{-1}$ ), simultânea e respectivamente, foram sucessivamente injetadas no sistema FIA com detecção amperométrica pulsada $(+0,9$ e $+1,35 \mathrm{~V})$ usando o sistema proposto. A Figura 3 apresenta os resultados obtidos sem (A) e com (B) o emprego de um pulso de potencial de limpeza $(0,0 \mathrm{~V} / 300 \mathrm{~ms})$.
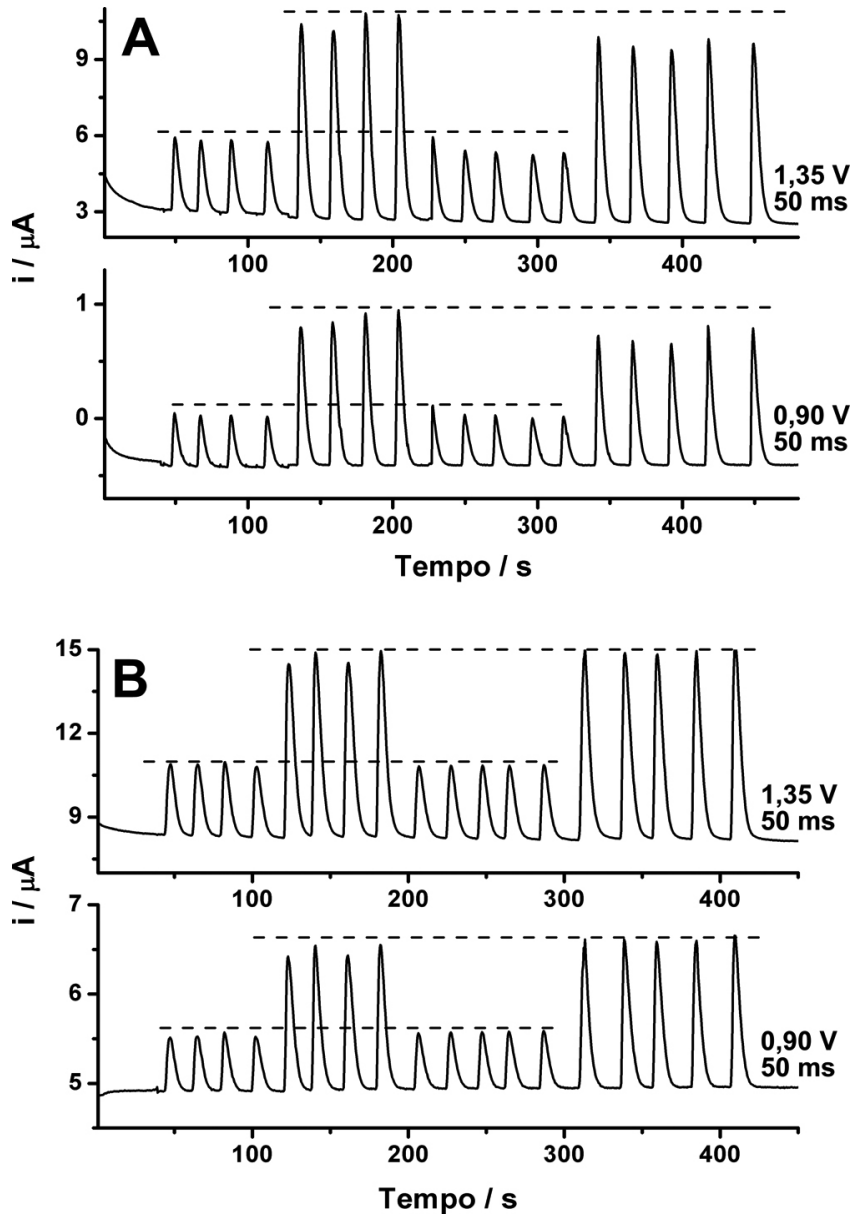

Figura 3. Amperogramas obtidos com o sistema FIA proposto em função de injeções sucessivas de soluções padrão contendo uma mistura de AA e AS em duas concentrações diferentes $\left(30+50\right.$ e $80+130 \mu \mathrm{mol} L^{-1}$ de AA+AS, respectivamente). (A) Sem aplicação do pulso de potencial de limpeza; (B) com aplicação do pulso de potencial de 0,0 V por $300 \mathrm{~ms}$ (amperograma não apresentado); vazão de 4,5 $\mathrm{mL} \mathrm{min}^{-1}$; volume injetado: $100 \mu \mathrm{L}$

Apesar do eletrodo de BDD apresentar boa resistência à desativação ou passivação ou envenenamento de sua superfície eletródica (além do curto período de contato entre o eletrodo e a solução contendo os analitos - sistema FIA), uma queda na corrente de oxidação dos compostos estudados foi verificada quando somente os dois pulsos de potenciais foram aplicados $(0,90$ e 1,35 V; Figura 3A). Como a técnica de amperometria de múltiplos pulsos (MPA) permite a aplicação de até 10 pulsos de potenciais sequenciais (tempo de aplicação de cada pulso na ordem de milissegundos) em função do tempo (software GPES - Metrohm Autolab), uma estratégia testada foi a inclusão de um terceiro pulso de potencial no sistema de detecção (com a função de limpeza eletroquímica da superfície do eletrodo periódica ou "constante"). A Figura 3B apresenta os resultados obtidos com injeções sucessivas das mesmas soluções do experimento da Figura $3 \mathrm{~A}$ com a inclusão de um terceiro pulso de potencial no sistema de detecção (0,90 V/50 ms; 1,35 V/50 ms V e 0,00 V/ 300 ms). Neste caso, é importante observar que o eletrodo de trabalho permanece 100 ms promovendo a oxidação de AA ou AA+AS (etapa de contaminação) e $300 \mathrm{~ms}$ promovendo a limpeza eletroquímica do eletrodo. Em outras palavras, o eletrodo permanecerá $25 \%$ do tempo na etapa de contaminação e $75 \%$ do tempo na etapa de limpeza eletroquímica. A opção pelo pulso de potencial de $0,0 \mathrm{~V}$ se deve ao fato de que neste potencial um sinal de redução ou dessorção é observado para o produto de oxidação do AS no experimento de voltametria cíclica 
Tabela 1. Desvios padrões relativos $(n=9)$ calculados a partir dos estudos de repetibilidade com o sistema FIA-MPA proposto, sem e com o emprego de um pulso de potencial de limpeza eletroquímica $(0,0 \mathrm{~V} / 300 \mathrm{~ms})$

\begin{tabular}{ccccc}
\hline \multirow{2}{*}{ Solução injetada (AA + AS)/ $\mu \mathrm{mol} \mathrm{L}^{-1}$} & \multicolumn{2}{c}{ Sem E de limpeza } & \multicolumn{2}{c}{ Com E de limpeza $(0,0 \mathrm{~V} / 300 \mathrm{~ms})$} \\
\cline { 2 - 5 } & $0,90 \mathrm{~V}$ & $1,35 \mathrm{~V}$ & $0,90 \mathrm{~V}$ & $1,35 \mathrm{~V}$ \\
\hline $30+50$ & $2,23 \%$ & $2,21 \%$ & $0,95 \%$ & $0,66 \%$ \\
$80+130$ & $2,91 \%$ & $2,15 \%$ & $1,48 \%$ & $1,09 \%$ \\
\hline
\end{tabular}

(Figura 1A). A Tabela 1 apresenta uma comparação entre os resultados obtidos nos estudos de repetibilidade sem e com o emprego de um pulso de potencial de limpeza.

Como se pode observar, obteve-se uma melhora considerável nos resultados após a inclusão do pulso de potencial que promove limpeza eletroquímica "constante" do eletrodo de trabalho (BDD). É importante salientar que o uso do pulso de potencial de limpeza não implica em perda de desempenho do método. Somente ocorre um aumento na corrente capacitiva devido ao incremento na amplitude dos pulsos de potenciais aplicados. A média $(\mathrm{n}=10)$ no ruído da linha base foi de 5,3 $\pm 0,6$ e 7,0 $\pm 1,0 \mathrm{nA}$ no experimento sem e com o uso do pulso de potencial de limpeza eletroquímica, respectivamente. Estes resultados demonstram que o ruído da linha base não variou significativamente com a inclusão do pulso de potencial de limpeza.

Foi realizado outro estudo com o objetivo de se identificar a faixa de concentração onde existe uma relação linear com a respectiva corrente de oxidação de cada composto. O AA apresentou uma faixa linear de resposta entre 10 e $120 \mu \mathrm{mol} \mathrm{L}^{-1} \mathrm{em}$ ambos os pulsos de potenciais e o AS entre 16 e $200 \mu \mathrm{mol} \mathrm{L}^{-1}$ no pulso de potencial de 1,35 V. Em todos os casos, o coeficiente de correlação foi igual ou melhor que 0,998 . Os estudos realizados para a identificação da faixa de concentração onde o sinal amperométrico de oxidação de AA é linear versus concentração também foram usados para averiguar em que faixa de concentração o fator entre a corrente de oxidação do AA em $+1,35$ e $+0,90 \mathrm{~V}\left(\mathrm{I}_{\mathrm{AA} \mathrm{em}+1,35 \mathrm{~V}} / \mathrm{I}_{\mathrm{AAem}+0,90 \mathrm{~V}}\right)$ é constante. $\mathrm{Na}$ faixa de concentração estudada (entre 10 e $120 \mu \mathrm{mol} \mathrm{L}^{-1}$ ), o valor médio do fator foi calculado em 1,05 $\pm 0,03(\mathrm{n}=5)$. Segundo estudos realizados, em cada nova calibração do método é aconselhável que uma solução contendo somente AA seja injetada para determinação do fator existente entre os sinais adquiridos nos pulsos de potenciais de $+0,90$ e $1,35 \mathrm{~V}$.

Três condições foram consideradas para se definir a faixa de concentração a ser usada na construção da curva de calibração, na análise simultânea de AA e AS em formulações farmacêuticas: faixa de concentração onde há linearidade entre a corrente de oxidação detectada e a concentração, tanto para AA como para AS; faixa de concentração de AA onde o fator de correção $\left(\mathrm{i}_{\mathrm{AA}+1,35 \mathrm{~V}} / \mathrm{i}_{\mathrm{AA}+0,90 \mathrm{~V}}\right)$ é constante; relação entre a concentração de AA e AS existente em formulações farmacêuticas comerciais (AA = $240 \mathrm{mg}$; AAS $=400$ mg; 1,67 vezes).

A Figura 4 apresenta os amperogramas obtidos nos pulsos de potenciais de $+0,90$ e $+1,35 \mathrm{~V}$ em função da injeção, em triplicata, de soluções padrão contendo, respectivamente, AA e AS: (a) 30 e 50; (b) 40 e 65; (c) 50 e 80; (d) 60 e 100; (e) 70 e 115; (f) 80 e 130 $\mu \mathrm{mol} \mathrm{L}{ }^{-1}$. Além disto, uma solução $(\mathrm{g})$ contendo somente $20 \mu \mathrm{mol} \mathrm{L} \mathrm{L}^{-1}$ de AA também foi injetada com o objetivo de se obter (calcular) o fator de correção a ser usado na Equação 3, para ter acesso à corrente proveniente da oxidação do AS no pulso de potencial de $1,35 \mathrm{~V}$.

As curvas analíticas apresentaram boa linearidade na faixa de concentração estudada e as equações correspondentes foram:

\footnotetext{
$\mathrm{I}_{\mathrm{AA}}=\mathrm{I}_{+0.9 \mathrm{~V}} ; \mathrm{I}(\mu \mathrm{A})=0,2568+0,0379 \mathrm{c}\left(\mu \mathrm{mol} \mathrm{L}^{-1}\right) ; \mathrm{r}=0,999$

$\mathrm{I}_{\mathrm{AS}}=\mathrm{I}_{+1,35 \mathrm{~V}}-\left(\right.$ fator $\left.\mathrm{x} \mathrm{I}_{+0,9 \mathrm{v}}\right) ; \mathrm{I}(\mu \mathrm{A})=0,2862+0,0623 \mathrm{c}\left(\mu \mathrm{mol} \mathrm{L}^{-1}\right)$; $r=0,999$
}

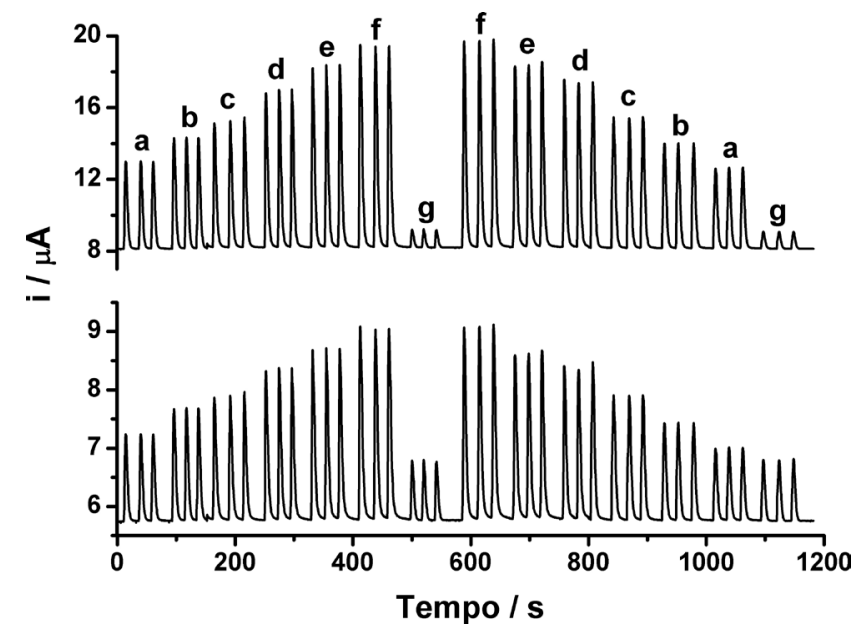

Figura 4. Amperogramas obtidos a partir de injeções de soluções padrão contendo, respectivamente, concentrações crescentes de AA + AS: (a) 30 e 50; (b) 40 e 65; (c) 50 e 80; (d) 60 e 100; (e) 70 e 115; (f) 80 e 130 rmol L $L^{-1}$;

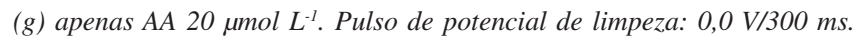
Vazão de 4,5 $\mathrm{mL} \mathrm{min}^{-1}$; volume de injeção: $100 \mu \mathrm{L}$

A partir destes dados, os limites de detecção (3 vezes o desvio padrão do branco $)^{26}$ foram calculados em 174 e $164 \mathrm{nmol} \mathrm{L}^{-1}$ de AA e AAS, respectivamente.

O desempenho do método foi verificado com a análise de 4 amostras de lotes diferentes de formulações farmacêuticas contendo AA e AAS. A Tabela 2 apresenta uma comparação entre os resultados obtidos usando o método proposto e os obtidos por HPLC.

Tabela 2. Comparação dos resultados obtidos na determinação simultânea de AA e AAS em quatro formulações farmacêuticas empregando o método proposto (FIA- MPA) e HPLC ( $\mathrm{n}=3$ )

\begin{tabular}{ccccccc}
\hline Amostra & Fármaco & Bula $^{(1)}$ & HPLC $^{(1)}$ & $\begin{array}{c}\text { FIA- } \\
\text { MPA }^{(1)}\end{array}$ & $\mathrm{E}_{1}(\%)$ & $\mathrm{E}_{2}(\%)$ \\
\hline 1 & AA & 240 & $233 \pm 3$ & $224 \pm 4$ & -7 & $-3,7$ \\
& AAS & 400 & $349 \pm 3$ & $364 \pm 6$ & -9 & 4,3 \\
2 & AA & 240 & $238 \pm 4$ & $229 \pm 7$ & $-4,5$ & $-3,7$ \\
& AAS & 400 & $385 \pm 5$ & $392 \pm 9$ & -2 & 2 \\
3 & AA & 240 & $280 \pm 4$ & $279 \pm 6$ & 16 & -1 \\
& AAS & 400 & $430 \pm 4$ & $430 \pm 3$ & 7,5 & 0 \\
4 & AA & 240 & $287 \pm 6$ & $287 \pm 6$ & 19,5 & 0 \\
& AAS & 400 & $430 \pm 5$ & $424 \pm 4$ & 6 & $-1,4$
\end{tabular}

(1) Unidade $=\mathrm{mg} / \mathrm{comprimido} ; \mathrm{E}_{1}=100 \times($ FIA-MPA - Valor da bula $) /$ Valor da bula; $\mathrm{E}_{2}=100 \times($ FIA-MPA - HPLC)/HPLC

O método proposto de determinação simultânea de AA e AAS apresentou resultados similares aos obtidos por HPLC. Segundo o teste $\mathrm{f}$, os dois métodos fornecem precisões equivalentes, considerando-se um nível de confiança de $95 \%$. Todos os valores calculados foram inferiores ao valor tabelado $\left(f_{\text {tabelado }}=9,28\right)$. Considerando o teste $t$, 
os resultados médios $(\mathrm{n}=3)$ de ambos os métodos não apresentaram diferença significativa em um nível de confiança de $95 \%$, sendo que o $t_{\text {calculado }}$ foi inferior ao $t_{\text {tabelado }}(2,78)$. Somente a amostra 1 apresentou um valor calculado superior ao valor tabelado. Além disto, estudos de adição e recuperação também foram realizados com o objetivo de verificar possíveis interferências geradas pela matriz dessas amostras sobre a resposta obtida. Neste estudo, um resultado médio de 104 $\pm 3 \%(\mathrm{n}=8)$ foi obtido, o que demonstra a ausência de problemas relacionados com a matriz da amostra.

\section{CONCLUSÕES}

O método proposto de determinação simultânea de ácido ascórbico (AA) e ácido acetilsalicílico (AAS) em formulações farmacêuticas usando análise por injeção em fluxo com detecção amperométrica de múltiplos pulsos (FIA-MPA) apresenta diversas características que podem ser consideradas favoráveis: baixo custo operacional, dissolução e simples diluição como etapas de pré-tratamento das amostras, alta frequência analítica (125 injeções $\mathrm{h}^{-1}$ ), emprego de reagentes de baixa toxicidade ( $\mathrm{NaOH}$ e tampão acetato) e geração de pequena quantidade de resíduos por análise ( $2 \mathrm{~mL}$ por injeção). Além disto, os resultados obtidos com o método proposto são similares aos obtidos por HPLC a um nível de confiança de $95 \%$.

\section{AGRADECIMENTOS}

Ao CNPq (476269/2010-5), à FAPEMIG (APQ-01430-11) e CAPES pelo suporte financeiro.

\section{REFERÊNCIAS}

1. Shriver, D. A.; Dove, P. A.; White, C. B.; Sandor, A.; Rosenthale, M. E.; Toxicol. Appl. Pharmacol. 1977, 42, 75.

2. Dammann, H. G.; Saleki, M.; Torz, M.; Schulz, H. U.; Krupp, S.; Schurer, M.; Timm, J.; Gessner, U.; Aliment. Pharmacol. Ther. 2004, 19 , 367.

3. Candelario-Jalil, E.; Akundi, R. S.; Bhatia, H. S.; Lieb, K.; Appel, K.; Munoz, E.; Hull, M.; Fiebich, B. L.; J. Neuroimmunol. 2006, 174, 39.

4. Supalkova, V.; Petrek, J.; Havel, L.; Krizkova, S.; Petrlova, J.; Adam, V.; Potesil, D.; Babula, P.; Beklova, M.; Horna, A.; Kizek, R.; Sensors 2006, 6, 1483 .

5. Thomis, R.; Roets, E.; Hoogmartens, J.; J. Pharm. Sci. 1984, 73, 1830; Akay, C.; Degim, I. T.; Sayal, A.; Aydin, A.; Ozkan, Y.; Gul, H.; Turk. J. Med. Sci. 2008, 38, 167; Kmetec, V.; J. Pharm. Biomed. Anal. 1992, $10,1073$.

6. Ustun, M.; Sungur, S.; Pharmazie 1992, 47, 459; Dinc, E.; Talanta 1999, 48, 1145; Sena, M. M.; Fernandes, J. C. B.; Rover, L.; Poppi, R. J.; Kubota, L. T.; Anal. Chim. Acta 2000, 409, 159; Dinc, E.; Ozdemir, A.; Pharmazie 2004, 59, 700; Dinc, E.; Baleanu, D.; Rev. Chim. 2008, 59, 499; Dinc, E.; Baleanu, D.; J. Braz. Chem. Soc. 2008, 19, 434.

7. de Barros, R. D. M.; Ribeiro, M. C.; An-Sumodjo, P. T.; Juliao, M. S. D.; Serrano, S. H. P.; Ferreira, N. G.; Quim. Nova 2005, 28, 317.

8. Rao, T. N.; Fujishima, A.; Diamond Relat. Mater. 2000, 9, 384; Compton, R. G.; Foord, J. S.; Marken, F.; Electroanalysis 2003, 15, 1349; Kawarada, H.; Surface Science Reports 1996, 26, 205; Hupert, M.; Muck, A.; Wang, R.; Stotter, J.; Cvackova, Z.; Haymond, S.; Show, Y.; Swain, G. M.; Diamond Relat. Mater. 2003, 12, 1940.

9. Oliveira, R. T. S.; Salazar-Banda, G. R.; Ferreira, V. S.; Oliveira, S. C.; Avaca, L. A.; Electroanalysis 2007, 19, 1189; Ribeiro, F. W. P.; Cardoso, A. S.; Portela, R. R.; Lima, J. E. S.; Machado, S. A. S.; de Lima-Neto, P.; De Souza, D.; Correia, A. N.; Electroanalysis 2008, 20, 2031; Souza,
C. D.; Braga, O. C.; Vieira, I. C.; Spinelli, A.; Sens. Actuators, B 2008, 135, 66; Sartori, E. R.; Medeiros, R. A.; Rocha-Filho, R. C.; FatibelloFilho, O.; J. Braz. Chem. Soc. 2009, 20, 360; Julião, M. S. D.; Almeida, E. C.; La Scalea, M. A.; Ferreira, N. G.; Compton, R. G.; Serrano, S. H. P.; Electroanalysis 2005, 17, 269; Andrade, L. S.; Rocha-Filho, R. C.; Cass, Q. B.; Fatibello-Filho, O.; Anal. Methods 2010, 2, 402; Lourenção, B. C.; Medeiros, R. A.; Rocha-Filho, R. C.; Fatibello-Filho, O.; Electroanalysis 2010, 22, 1717; Andrade, L. S.; Rocha-Filho, R. C.; Cass, Q. B.; Fatibello-Filho, O.; Electroanalysis 2009, 21, 1475; Lourenção, B. C.; Medeiros, R. A.; Rocha-Filho, R. C.; Mazo, L. H.; Fatibello-Filho, O.; Talanta 2009, 78, 748; de Lima-Neto, P.; Correia, A. N.; Portela, R. R.; Julião, M. D.; Linhares, G. F.; de Lima, J. E. S.; Talanta 2010, 80, 1730; Sartori, E. R.; Medeiros, R. A.; Rocha-Filho, R. C.; Fatibello-Filho, O.; Talanta 2010, 81, 1418; Batista, E. F.; Sartori, E. R.; Medeiros, R. A.; Rocha-Filho, R. C.; Fatibello-Filho, O.; Anal. Lett. 2010, 43, 1046; Martins, I.; Canaes, L. D.; Doretto, K. M.; Rath, S.; Electroanalysis 2010, 22, 455; Martins, I.; Carreira, F. C.; Canaes, L. S.; Campos, F. A. D.; Cruz, L. M. D.; Rath, S.; Talanta 2010, 85, 1; Santos, K. D.; Braga, O. C.; Vieira, I. C.; Spinelli, A.; Talanta 2010, 80, 1999.

10. dos Santos, W. T. P.; Gimenes, D. T.; Richter, E. M.; Angnes, L.; Quim. Nova 2011, 34, 1753.

11. dos Santos, W. T. P.; Gimenes, D. T.; de Almeida, E. G. N.; Eiras, S. D.; Albuquerque, Y. D. T.; Richter, E. M.; J. Braz. Chem. Soc. 2009, 20, 1249; Medeiros, R. A.; Lourenção, B. C.; Rocha-Filho, R. C.; Fatibello-Filho, O.; Anal. Chem. 2010, 82, 8658; dos Santos, W. T. P.; de Almeida, E. G. N.; Ferreira, H. E. A.; Gimenes, D. T.; Richter, E. M.; Electroanalysis 2008, 20, 1878.

12. Silva, R. A. B.; Gimenes, D. T.; Tormin, T. F.; Munoz, R. A. A.; Richter, E. M.; Anal. Methods 2011, 3, 2804.

13. Tormin, T. F.; Gimenes, D. T.; Silva, L. G.; Ruggiero, R.; Richter, E. M.; Ferreira, V. S.; Munoz, R. A. A.; Talanta 2010, 82, 1599; Gimenes, D. T.; dos Santos, W. T. P.; Tormin, T. F.; Munoz, R. A. A.; Richter, E. M.; Electroanalysis 2010, 22, 74.

14. Gimenes, D. T.; de Freitas, J. M.; Munoz, R. A. A.; Richter, E. M.; Electroanalysis 2011, 23, 2521.

15. Gimenes, D. T.; dos Santos, W. T. P.; Munoz, R. A. A.; Richter, E. M.; Electrochem. Commun. 2010, 12, 216.

16. Enache, T. A.; Fatibello-Filho, O.; Oliveira-Brett, A. M.; Comb. Chem. High Throughput Screening 2010, 13, 569.

17. Franeta, J. T.; Agbaba, D.; Eric, S.; Pavkov, S.; Aleksic, M.; Vladimirov, S.; Farmaco 2002, 57, 709.

18. Bergamin, H.; Zagatto, E. A. G.; Krug, F. J.; Reis, B. F.; Anal. Chim. Acta 1978, 101, 17.

19. Silva, W. C.; Pereira, P. F.; Marra, M. C.; Gimenes, D. T.; Cunha, R. R.; Silva, R. A. B.; Munoz, R. A. A.; Richter, E. M.; Electroanalysis 2011, 23, 2764.

20. Bart, M.; van Os, P.; Kamp, B.; Bult, A.; van Bennekom, W. P.; Sens. Actuators, B 2002, 84, 129.

21. Pedrotti, J. J.; Angnes, L.; Gutz, I. G. R.; Electroanalysis 1996, 8, 673.

22. Terashima, C.; Rao, T. N.; Sarada, B. V.; Kubota, Y.; Fujishima, A.; Anal. Chem. 2003, 75, 1564.

23. Salazar-Banda, G. R.; Andrade, L. S.; Nascente, P. A. P.; Pizani, P. S.; Rocha-Filho, R. C.; Avaca, L. A.; Electrochim. Acta 2006, 51, 4612.

24. dos Santos, W. T. P.; Ceolin, M. P.; de Albuquerque, Y. D. T.; Richter, E. M.; Quim. Nova 2007, 30, 1754.

25. Torriero, A. A. J.; Luco, J. M.; Sereno, L.; Raba, J.; Talanta 2004, 62, 247.

26. Miller, J. C.; Miller, J. N.; Statistics for Analytical Chemistry, Harwood: Chichester, 1992. 